\title{
Morts cellulaires et système immunitaire
}

La mise en place et le fonctionnement du système immunitaire impliquent des processus de mort cellulaire dont les lymphocytes $T$ sont soit l'objet, soit l'agent. L'élimination des lymphocytes $T$ potentiellement nocifs, dotés de récepteurs capables de reconnaître des antigènes du soi, se fait par un mécanisme de suicide induit de type « apoptose ». Certains des lymphocytes survivants, une fois leur maturation achevée, vont être capables de cytotoxicité envers des cellules du même organisme présentant à leur surface des antigènes étrangers. Les mécanismes de l'apoptose et de la cytotoxicité des cellules $T$ sont encore mal connus; ils pourraient être élucidés grâce à l'apport conjoint des techniques de clonage moléculaire et de génétique somatique.

\section{Pierre Golstein}

\section{RÉFÉRENCES}

l. MacDonald HR, Howe RC, Pedrazzini T, et al. T-cell lineages, repertoire selection and tolerance induction. Immunological Rev $1988 ; 104: 157-82$

2. Von Boehmer H, Teh HS, Kisielow P. The thymus selects the useful, neglects the useless and destroys the harmful. Immunol Today $1989 ; 10: 57-61$

3. Catsicas S, Thanos S, Clarke PGH. Major role for neuronal death during brain development : refinement of topographical connections. Proc Natl Acad Sci USA 1987; 84 8165-8.

4. Smith CA, Williams GT, Kingston R, Jenkinson EJ, Owen JJT. Antibodies to CD3/T cell receptor complex induce death by apoptosis in immature $T$ cells in thymic cultures. Nature 1989 ; 3.97 : 181-4.

5. Mercep C, Bluestone JA, Noguchi PD, Ashwell JD. Inhibition of transformed T cell growth in vitro by monoclonal antibodies directed against distinct activating molecules. J Immunol 1988 ; 140 : 324-35.

\section{ADRESSE}

P. Golstein : directeur de recherche à l'Inserm, centre d'immunologie Inserm-Cnrs de Marseille-Luminy, Case 906, 13288 Marseille

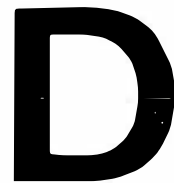

ans le système immunitaire, comme partout dans un individu multicellulaire, la mort cellulaire n'est pas un phénomène fortuit : elle est programmée, sans doute autant que multiplication et migration cellulaires. Multiplication, migration et mort constituent en fait les étapes majeures d'un destin cellulaire. Ces étapes sont toutes trois nécessaires au développement normal de tissus, organes, et finalement organismes. Hors sénescence ou pathologie, la mort cellulaire est donc bénéfique à l'individu. Essentielle et positive, la mort cellulaire en est d'autant plus, toute réticence culturelle levée, « dicible».

Cette mort cellulaire peut prendre deux aspects en ce qui concerne le système immunitaire: mort cellulaire dans le système immunitaire, et il s'agit de lymphocytes qui meurent; mort cellulaire par le système immunitaire, et il s'agit alors de lymphocytes qui tuent. Nous allons considérer successivement ces deux aspects, différents par leurs circonstances de survenue, mais peut-être apparentés dans leur mécanisme et dans leur finalité.

\section{Mort cellulaire dans le système immunitaire}

Il s'agit d'une mort cellulaire survenant physiologiquement lors d'une étape normale du développement du système immunitaire. La finalité semble en être la réduction sélective du répertoire (le répertoire étant l'ensemble des récepteurs, capables de reconnaître des antigènes, présents à la surface des lymphocytes). Un excellent exemple en est fourni par le thymus. Les lymphocytes pénètrent dans le thymus à l'état de cellules précurseurs qui, à la suite d'une efficace mais aveugle génération de diversité, possèdent des récepteurs «réarrangés » au hasard, dirigés contre n'importe quel antigène, y compris les antigènes du soi. Ce résultat d'une hyperproduction incontrôlée doit être corrigé pour en éliminer en particulier les lymphocytes dotés de récepteurs anti-soi. L'élimination en question est effectuée par une mort cellulaire également très importante quantitative- 
ment, mais, elle, sélective [1, 2]. Cette sélection négative par mort cellulaire, entraînant une délétion clonale, s'accompagne d'une sélection positive que nous ne considérerons pas ici. Le thymus, ainsi, «sélectionne l'utile... et détruit le nuisible » [2]. Ne ressortent finalement du thymus pour aller coloniser les organes lymphoïdes secondaires qu'un tout petit pourcentage des cellules qui y sont entrées.

La mort cellulaire élimine donc, dans le système immunitaire, parmi tous les lymphocytes produits, ceux qui portent des récepteurs inappropriés. Ce processus darwinien auquel la mort cellulaire contribue se retrouve ailleurs dans l'organisme, avec parfois des similitudes frappantes (par exemple, selon certains travaux récents [3], la mort cellulaire éliminerait, dans le système nerveux, parmi tous les neurones mis en place, ceux qui ont établi des connexions inappropriées - ou pas de connexions du tout).

Quelques modèles expérimentaux nous révèlent certains des traits les plus saillants de l'induction d'une telle mort cellulaire développementale dans le système immunitaire.

Ainsi dans le laboratoire de John Owen, à Birmingham, des thymocytes de souris foetales, cultivés in vitro, ont été soumis à des anticorps anti-CD3 (dirigés contre le complexe récepteur pour l'antigène de lymphocytes T). Un groupe témoin était constitué de thymocytes incubés sans anticorps. Au bout de 18 heures d'incubation, alors que pratiquement $100 \%$ des cellules du groupe témoin survivaient, plus de la moitié des cellules soumises aux anticorps antiCD3 étaient mortes [4], avec un aspect morphologique caractéristique (voir plus bas), mimant sans doute ainsi in vitro le processus de sélection par délétion clonale survenant dans le thymus. Il est à noter que la stimulation, par l'intermédiaire de son récepteur pour l'antigène, d'un lymphocyte à un stade plus tardif de maturation aboutit non pas à sa mort mais à sa multiplication. Suivant le stade de développement d'un lymphocyte, la même stimulation de son récepteur de surface aboutit ainsi à des situations très différentes.

Dans le laboratoire de J. Ashwell, au
$\mathrm{NIH}$, des hybridomes $\mathrm{T}$ antigènespécifiques ont été soumis aux mêmes anticorps anti-CD3. Cette stimulation entraîne d'une part l'activation de ces cellules objectivée par la production temporaire d'interleukine, et d'autre part leur mort en quelques heures [5].

Enfin, dans le laboratoire de Ian MacLennan, à Birmingham, des lymphocytes B provenant d'amygdales humaines sont mis en culture in vitro. Spontanément, $90 \%$ de ces cellules vont mourir au bout de 24 heures d'incubation, en présentant un aspect caractéristique de mort cellulaire développementale. Si on ajoute à ces cellules, en début d'incubation, des anticorps antiimmunoglobulines de surface, ces cellules survivent pratiquement toutes (Liu et al., sous presse). Ici, la stimulation du récepteur pour l'antigène sauve les lymphocytes d'une mort programmée. Cela n'est qu'en apparence en contradiction avec les expériences précédentes; en effet, le point commun est la relation entre transduction d'un signal par le récepteur spécifique pour l'antigène et mort cellulaire, induite ou bloquée.

Au total, ces expériences, parmi d'autres, montrent que la stimulation du récepteur pour l'antigène peut : soit induire une multiplication lymphocytaire, dans les lymphocytes mûrs ; soit induire une mort programmée, dans les thymocytes immatures, ou dans certains hybridomes $T$ (pour ces derniers, nous avons vu que la mort cellulaire peut être précédée de signes d'activation cellulaire comme la production d'interleukines; nous verrons plus loin que la mort de thymocytes s'accompagne d'étapes biosynthétiques témoignant également d'une activation cellulaire); soit prévenir une mort programmée, comme dans les lymphocytes B mis en culture.

Nous avons largement utilisé, pour qualifier la mort cellulaire développementale, le terme de mort programmée. Ce terme est cependant ambigu, recouvrant deux notions distinctes.

Tout d'abord il s'agit d'une mort programmée dans l'organisme; comme nous venons de le voir, elle survient dans certains organes, à certains stades du développement, pour certains types cellulaires. Cette survenue programmée a suggéré à Kerr $e t$ al. pour ce phénomème le nom d'apoptose [6], par allusion à cette mort programmée que constitue à l'évidence la chute automnale des feuilles d'un arbre.

Mais également, il s'agit d'une mort programmée dans la cellule, avec une cascade d'événements comprenant, après induction et transduction du signal correspondant, une série d'étapes intracellulaires. Ce déroulement programmé comporte, sur le plan morphologique [6, 7], une condensation cytoplasmique, comme s'il y avait fuite d'eau vers l'espace extracellulaire, avec de profondes invaginations donnant un aspect de bullage de surface (figure 1 . p. 548). Parallèlement, il y a condensation de l'ADN nucléaire sous forme de masses visibles sous la membrane nucléaire, à la périphérie du noyau. Ces phénomènes s'accompagnent, sur un plan moléculaire, d'une frappante dégradation de l'ADN, en fragments de 180 à 200 paires de bases correspondant sensiblement à la taille d'un nucléosome, suggérant l'intervention précoce d'endonucléases. La fragmentation de l'ADN a été notée depuis déjà 15-20 ans dans des systèmes expérimentaux très divers de mort cellulaire (voir [8-10] et les références y incluses; et la description très récente et fascinante d'un anticorps monoclonal, reconnaissant une structure de membrane différente de celles connues jusqu'à présent, et déclenchant ainsi la mort cellulaire [11]); cependant, la plupart de ces études ont porté sur des cellules lymphoïdes, tuées par irradiation [12-14] ou par incubation en présence de glucocorticoïdes [15-17] ou d'anticorps anti-CD3 [4, 5, 19].

Une phase ultérieure de ce processus consiste en une fragmentation cellulaire massive, avec génération de fragments cellulaires dont chacun reste entouré de membrane cytoplasmique et contient du cytoplasme et des fragments de noyaux. Ces fragments cellulaires seront ensuite rapidement phagocytés.

Fait intéressant, cette mort cellulaire peut être inhibée par des inhibiteurs de synthèse de macromolécules, par exemple des inhibiteurs de synthèse d'ARN comme l'actinomycine D, ou de synthèse protéique comme la 
6. Kerr JFR, Wyllie AH, Currie AR. Apoptosis: a basic biological phenomenon with wide-ranging implications in tissue kinetics. Br J Cancer 1972; 26: 239-57.

7. Duvall E, Wyllie AH. Death and the cell. Immunol Today $1986 ; 7$ : 115-9.

8. Williamson R. Properties of rapidly labelled deoxyribonucleic acid fragments isolated from the cytoplasm of primary cultures of embryonic mouse liver cells. J Mol Biol 1970 ; $51: 157-68$.

9. Williams JR, Little JB, Shipley WU. Association of mammalian cell death with a specific endonucleolytic degradation of DNA Nature 1974 ; 252 : 754-5.

10. Appleby DW, Modak SP. DNA degradation in terminally differentiating lens fiber cells from chick embryos. Proc Natl Acad Sci USA 1977; 74 : 5579-83.

11. Trauth BC, Klas C, Peters AMJ, et al. Monoclonal antibody-mediated tumor regression by induction of apoptosis. Science 1989 ; $245: 301-5$.

12. Skalka M, Matyasova J, Cejkova M. DNA in chromatin of irradiated lymphoid tissues degrades in vivo into regular fragments. FEBS Lett 1976; 72 : 271-4.

13. Sellins KS, Cohen JJ. Gene induction by gamma-irradiation leads to DNA fragmentation in lymphocytes. J Immunol 1987; 139 . 3199-206.

14. Yamada T, Ohyama H. Radiation-induced interphase death of rat thymocytes is internally programmed (apoptosis). Int J Radiat Biol 1988; 53 : 65-75.

15. Wyllie AH. Glucocorticoid-induced thymocyte apoptosis is associated with endogenous endonuclease activation. Nature 1980 284: 555-6.

16. Cohen JJ, Duke RC. Glucocorticoid activation of a calcium-dependent endonuclease in thymocyte nuclei leads to cell death. $J$ Immunol 1984; 132 : 38-42.

17. Wyllie AH, Morris RG, Smith AL, Dunlop D. Chromatin cleavage in apoptosis : association with condensed chromatin morphology and dependence on macromolecular synthesis. J Pathol 1984; 142: 67-77.

18. Smith CA, Williams GT, Kingston $R$, Jenkinson EJ, Owen JJT. Apoptosis. Nature $1989 ; 338: 10-20$.

19. Shi Y, Sahai BM, Green DR. Cyclosporin $A$ inhibits activation-induced cell death in T-cell hybridomas and thymocytes. Nature $1989 ; 339: 625-6$.

20. Martin DP, Schmidt RE, DiStefano PS Lowry OH, Carter JG, Johnson EM Jr. Inhibitors of protein synthesis and RNA synthesis prevent neuronal death caused by nerve growth factor deprivation. J Cell Biol 1988 106: 829-44.

21. Kosaka H, Ogata M, Hikita I, et al. Model for clonal elimination in the thymus. Proc Natl Acad Sci USA 1989; 86 : 3773-7. 22. Pierce GB, Lewellyn AL, Parchment RE. Mechanism of programmed cell death in the blastocyst. Proc Natl Acad Sci USA 1989; 86 : 3654-8.

23. Sellins KS, Cohen JJ. Polyomavirus DNA is damaged in target cells during cytotoxic T-lymphocyte-mediated killing. J Virol

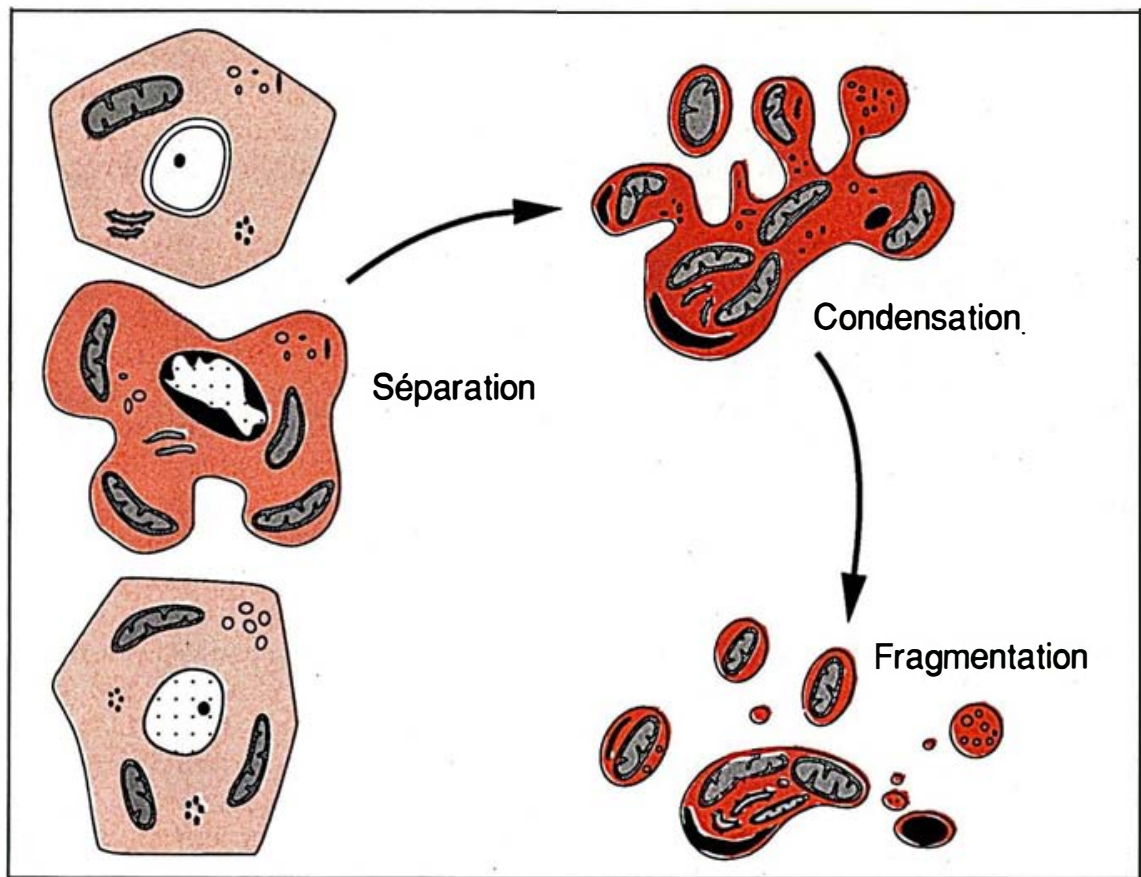

Figure 1. Mort cellulaire par apoptose d'une cellule d'un parenchyme. (D'après JFR Kerr et al. Br J Cancer $1972 ; 26$ : 239-57.)

cycloheximide $[13,14,16,17,20]$. La cellule qui meurt doit donc synthétiser certaines macromolécules pour mourir, elle participe activement à sa propre mort. C'est en ce sens qu'on a pu parler d'un suicide. De plus, comme nous l'avons vu, cette mort cellulaire n'est pas fortuite, mais programmée, induite; c'est en ce sens qu'on a pu parler de suicide induit. Lors de cette mort, on peut distinguer encore d'autres événements métaboliques ou anatomiques, que nous ne détaillerons pas ici.

Au total, quatre notions particulièrement génératrices de questions, voire d'expériences, se dégagent de ce qui précède.

- La mort cellulaire développementale est programmée quant à sa survenue et quant à son déroulement, dans le système immunitaire comme ailleurs dans l'organisme. Cependant, fait extrêmement rare pour un événement développemental, l'inducteur en est ici connu : il s'agit de l'antigène, dont le récepteur transduit cette induction.

- Au moins dans le système immunitaire, la conséquence de la transduction du signal inducteur est une activation conduisant soit à la mort soit à la multiplication cellulaire. Il n'y a donc pas ici de signal de mort auquel toute cellule répondrait de façon univoque. Le type de réponse de la cellule pourrait être fonction de son stade de développement : ce ne serait qu'à un stade précis de développement de la cellule que pourrait être mis en route un programme intracellulaire particulier, déclenché par un signal de surface donné, et aboutissant à la mort cellulaire. Alternativement, le type de réponse de la cellule pourrait être fonction de la réception simultanée d'un autre signal, fourni ou non par l'environnement; ainsi, la mort de certains hybridomes $\mathbf{T}$ nécessite non seulement qu'ils reconnaissent leur antigène cellulaire, mais aussi qu'ils soient exposés à un facteur soluble fabriqué par des cellules thymiques stromales [21]; de façon intéressante, des signaux du même ordre, reconnaissance cellule/cellule et facteur soluble, pourraient être impliqués lors d'un processus de mort cellulaire programmée chez l'embryon [22].

- Lors du déroulement de ce programme de mort cellulaire se produisent des événements morphologiques 
ou métaboliques. Parmi ceux-ci, lesquels sont des épiphénomènes et lesquels sont des maillons d'une chaîne causale aboutissant à la mort ? Surtout, parmi ces différents maillons, où est la première étape irréversible ? L'identification de cette première étape irréversible peut contribuer à une définition de la mort, dont l'élaboration est un problème ardu même à cette échelle cellulaire.

- Cette cascade d'événements comprend en particulier une ou plusieurs étapes de synthèse de macromolécules. Certaines de ces macromolécules sont nécessaires à la mort de la cellule qui les produit. Il sera évidemment fascinant de connaître la nature de ces molécules, que plusieurs équipes tentent actuellement d'isoler et d'identifier par clonage.

\section{Mort cellulaire par le système immunitaire}

Il s'agit d'une mort cellulaire fonctionnelle: le fait de tuer d'autres cellules constitue en effet l'une des fonctions du système immunitaire. Pour exercer cette fonction, le système immunitaire est richement pourvu en différents types de cellules tueuses, reconnaissant leurs cibles par différents récepteurs de surface : lymphocytes $T$ (avec leurs récepteurs spécifiques pour l'antigène), cellules $\mathrm{K}$ (avec des récepteurs pour des anticorps, eux-mêmes reconnaissant les cibles), cellules natural killer ou NK et cellules natural cytotoxic ou NC (ne nécessitant pas d'immunisation délibérée, lysant surtout des cellules tumorales, avec une spécificité encore mal définie), cellules LAK (lymphocytes activés par des lymphokines), macrophages plus ou moins activés, polynucléaires... Nous ne considérerons pas en détail ces différents types cellulaires ; l'essentiel de ce qui suit portera sur les lymphocytes $T$ cytotoxiques.

La finalité de la lyse par les lymphocytes ou cellules $T$ cytotoxiques est la mort de cellules présentant des molécules du «non-soi ». Non-soi dans ce cas peut être par exemple un virus ou un autre micro-organisme. Hébergé dans une cellule, le micro-organisme est hors d'atteinte du système immunitaire, sauf précisément des cellules $\mathrm{m} / \mathrm{s} n^{\circ} 8$ vol. 5 , octobre 89 cytotoxiques: la destruction de la cellule hôte détruit le micro-organisme, ou l'expose à d'autres agents effecteurs. L'intervention des cellules $T$ cytotoxiques dans la lutte antivirale pourrait inclure la destruction du virus dans les cellules cibles par fragmentation de l'ADN viral. Celle-ci pourrait se produire, soit de façon contemporaine à la fragmentation de l'ADN des cellules cibles (voir plus bas ; [23]), soit, par hypothèse, sans même qu'il y ait lyse des cellules cibles [24].

Le mécanisme de la cytotoxicité par cellules $T$ est étudié de façon très analytique, très réductionniste, dans des systèmes modèles in vitro.

Très schématiquement, ce mécanisme se décompose en trois phases : tout d'abord une phase de reconnaissance, correspondant à l'engagement de récepteurs et de ligands à la surface respectivement de cellules tueuses et cellules cibles; ensuite une phase dite de « coup mortel » correspondant à une lésion irréversible infligée par la cellule tueuse à la cellule cible; enfin une phase de désintégration de la cible, survenant même si la tueuse a quitté la cible. La rapidité de ces phénomènes est très remarquable; en particulier phase de reconnaissance et phase de coup mortel se produisent en quelques minutes voire quelques secondes; en revanche, la phase de désintégration de la cible peut durer plus longtemps, quelques minutes à quelques heures.

Nous ne décrirons pas en détail les aspects morphologiques de la lyse, qui ont été résumés récemment (voir en particulier [25]). Nous discuterons les principales hypothèses concernant le mécanisme moléculaire de la cytolyse, en particulier à l'étape de coup mortel, et notre approche expérimentale de ce mécanisme.

Deux hypothèses principales sont actuellement à la mode. La première fait intervenir une exocytose granulaire. En effet, le contact à la phase de reconnaissance entre tueuse et cible déclenche dans certaines conditions l'exocytose, à l'interface entre cellules tueuses et cellules cibles, de granules particuliers contenus dans la cellule tueuse. Les granules libèrent alors leur contenu en regard de la membrane de la cellule cible. Ce contenu comprend en particulier des molécules de perforine, structure récemment clonée [26-29], homologue de la molécule C9 de la cascade du complément. La perforine est capable de former canal en se polymérisant dans la membrane cellulaire. De tels canaux se formeraient dans la membrane de la cellule cible, entraînant la mort de celle-ci par déséquilibre osmotique (figure 2, p. 551).

Malgré le caractère séduisant de cette hypothèse, de nombreuses objections peuvent lui être opposées, comme l'existence de cellules tueuses dépourvues d'activité perforine détectable (quoique exprimant apparemment les transcrits correspondants, C. Nagler-Anderson et al., sous presse) ou capables de lyser des cibles en l'absence de $\mathrm{Ca}^{++}$extracellulaire, alors que l'action de la perforine est strictement dépendante du calcium; ces objections ont été récemment rappelées [25]. Par ailleurs, des molécules solubles autres que la perforine ont été proposées comme susceptibles de jouer un rôle dans la lyse, en particulier des molécules elles-mêmes dotées de propriétés lytiques comme le tumor necrosis factor ou TNF $\alpha$, la lymphotoxine ou TNF $\beta$ (quoique ces molécules ne rendent certainement pas compte de l'ensemble de la cytotoxicité T [30]) et une molécule apparentée aux TNF appelée leukalexine. Des arguments en faveur d'un mécanisme membranaire de la cytolyse ont été également avancés [31]. Signalons, dans ce contexte, les démonstrations récentes de formes de TNF $\alpha$ présentes à la membrane de cellules tueuses[32-35] qui peuvent être impliquées dans la variété particulière de cytotoxicité à médiation cellulaire appelée cytotoxicité NC.

La deuxième hypothèse majeure quant au mécanisme possible de la cytotoxicité à médiation cellulaire $T$ trouve son origine dans l'intéressante observation suivante. Des cellules cibles lysées par des cellules tueuses meurent morphologiquement comme des cellules en mort développementale, pour autant qu'on puisse en juger par les images de condensation et de fragmentation obtenues en microscopie électronique [36, 37], et par l'existence d'une fragmentation de l'ADN [38, 39]. Cela conduit à penser que la cellule tueuse 


\section{RÉFÉRENCES}

24. Martz E, Howell DM. CTL : virus control cells first and cytolytic cells second? DNA fragmentation, apoptosis and the prelytic halt hypothesis. Immunol Today 1989; 10 : 79-86

25. Golstein P, Brunet JF; Denizot F. Les cellules T tueuses. La Recherche 1988 ; 19 : 318 25.

26. Shinkai Y, Takio K, Okumura K. Homo logy of perforin to the ninth component of complement (C9). Nature 1988 ; 334 : 525-7.

27. Lichtenheld MG, Olsen KJ, Lu P, et al. Structure and function of human perforin. Nature 1988 ; 335 : 448-51.

28. Lowrey DM, Aebischer T, Olsen K, et al. Cloning, analysis, and expression of murine perforin 1 cDNA, a component of cytolytic T cell granules with homology to complement component C9. Proc Natl Acad SciUSA 1989 $86: 247-51$.

29. Kwon BS, Wakulchik M, Liu CC, et al. The structure of the mouse lymphocyte poreforming protein perforin. Biochem Biophys Res Commun 1989; 158: 1-10.

30. Jongeneel CV, Nedospasov SA, Plae tinck G, Naquet $P$, Cerottini JC. Expression of the tumor necrosis factor locus is no necessary for the cytolytic activity of $\mathrm{T}$ lymphocytes. J Immunol 1988 ; 140 : 1916-22.

31. Berke G. The cytolytic T lymphocyte and its mode of action. Immunol Lett 1989; 20 . 169-78.

32. Decker T, Lohmann-Matthes ML, Gifford GE. Cell-associated tumor necrosis factor (TNF) as a killing mechanism of activated cytotoxic macrophages. J Immunol 1987 138: 957-62

33. Ichinose $Y$, Bakouche $O$, Tsao JY, Eidler IJ. Tumor necrosis factor and IL-I associated with plasma membranes of activated human monocytes lyse monokine-sensitive but not monokine-resistant tumor cells whe reas viable activated monocytes lyse both. J Immunol $1988 ; 141$ : 512-8.

34. Kriegler M, Perez C, DeFay K, Albert I, Lu SD. A novel form of TNF/cachectin is a cell surface cytotoxic transmembrane protein : ramifications for the complex physiology of TNF: Cell 1988; $53: 45-53$.

35. Liu CC, Detmers PA, Jiang S Young JDE. Identification and characterization of a membrane-bound cytotoxin of murine cytolytic lymphocytes that is related to tumor necrosis factor/cachectin. Proc Natl Acad Sci USA 1989; 86 : 3286-90.

36. Don MM, Ablett G, Bishop CJ, et al. Death of cells by apoptosis following attachment of specifically allergized lymphocytes in vitro. Austr J Exp Biol 1977 ; 55 : 407-17.

37. Stacey NH, Bishop CJ, Halliday JW, et al. Apoptosis as the mode of cell death in antibody-dependent lymphocytotoxicity. J Cell Sci 1985; 74 : 169-79.

38. Russell JH. Internal disintegration model of cytotoxic lymphocyte-induced target damage. Immunological Rev 1983; 72 : 97-118.

39. Duke RC, Chervenak R, Cohen JJ. Endogenous endonuclease-induced DNA fragmentation : an early event in cell-mediated cytolysis. Proc Natl Acad Sci USA 1983 ; 80 : 6361. déclenche dans la cellule cible une cascade d'événements, très comparable à celle qui est observée lors d'une mort développementale, aboutissant à la lyse de cette cellule cible [36, 38]. Cependant, si la lyse de la cellule cible dans ces conditions possède les caractères morphologiques d'une mort développementale, elle n'en possède pas tous les caractères; en particulier, cette lyse n'est pas inhibée par des inhibiteurs de biosynthèse de macromolécules [39, 40]. En réponse à cette discordance, une hypothèse unificatrice a été proposée par Kerr et al. [41]: les macromolécules qui sont normalement fabriquées par la cellule qui meurt pour contribuer à sa propre lyse seraient, là, fabriquées par la cellule tueuse (figure 3, p. 552); la cellule tueuse mettrait la cellule cible en présence de ces molécules, permettant ainsi la continuation à l'intérieur de la cible de la cascade d'événements aboutissant à sa mort. Les lymphocytes $\mathrm{T}$ cytotoxiques auraient ainsi détourné à leur profit une étape du processus de suicide programmé, pour en faire un instrument de lyse. Cette hypothèse, aussi séduisante soit-elle, n'est pas démontrée ; et elle aurait à rendre compte de la lyse, ou de l'absence de lyse, des cellules tueuses elles-mêmes. D'autre part, nous avons discuté jusqu'à présent de la nature du mécanisme de lyse comme si ce mécanisme était unique. En fait, la multiplicité des molécules déjà connues et capables de propriétés lytiques, et un certain nombre de caractéristiques fonctionnelles, suggèrent que plusieurs mécanismes de lyse pourraient coexister, dans des cellules cytotoxiques différentes voire dans la même cellule cytotoxique. Un argument expérimental direct en faveur de cette dernière possibilité est fourni par le travail de Lancki et al. [42]. Ces auteurs ont utilisé un clone de lymphocytes $T$ capable de trois effets : (1) la lyse des cibles cellulaires nucléées spécifiques; (2) la lyse de globules rouges recouverts d'anticorps anti-CD3 ; et (3) la libération dans le milieu extracellulaire de sérine-estérases (reflétant l'exocytose du contenu granulaire) lors de chacun de ces deux types de lyse. Fait intéressant, l'addition de ciclosporine A inhibe la lyse des globules rouges et la libération d'estérase, mais n'inhibe pas la lyse de cellules cibles nucléées. Ces résultats suggèrent, en conformité avec des résultats antérieurs, que la ciclosporine A est capable d'inhiber en particulier la sécrétion par exocytose à partir de lymphocytes. Surtout, ces résultats suggèrent que deux mécanismes de lyse coexistent dans le clone considéré, une lyse de globules rouges, sensible à la ciclosporine $\mathrm{A}$, corrélée avec un phénomène d'exocytose (et peut-être liée à la libération de perforine) ; et une lyse de cellules cibles nucléées, insensible à la ciclosporine $\mathrm{A}$, qui ne semble pas nécessiter d'exocytose (donc semblant refléter l'existence d'un autre mécanisme indépendant de l'exocytose et de la perforine).

Il est intéressant de noter au passage que la ciclosporine $A$ inhibe la mort de thymocytes[19] et d'hybridomes T [43] stimulés par l'intermédiaire de leur récepteur pour l'antigène, mais n'inhiberait pas la mort cellulaire induite par d'autres agents (J.J. Cohen et al., résultats non publiés). De même, la ciclosporine A ne serait capable d'inhiber, parmi les différents modes de cytolyse par cellules $T$, que ceux qui dépendent du $\mathrm{Ca}^{++}$[44]. Un puint d'impact de la ciclosporine A pourrait ainsi être une étape $\mathrm{Ca}^{++}$-dépendante de l'activation consécutive à une stimulation du récepteur des cellules $\mathrm{T}$; cette étape serait elle-même située en amont d'événements tueurs dans un lymphocyte $\mathrm{T}$ cytotoxique, ou d'événements de lyse dans un lymphocyte $T$ qui meurt.

Au total, nous nous trouvons confrontés à plusieurs mécanismes possibles pour la cytotoxicité par lymphocytes $\mathrm{T}$, et à plusieurs molécules candidates à jouer un rôle dans ces mécanismes. Rien ne prouve actuellement que certaines de ces molécules rendent compte de certains de ces mécanismes, ni d'ailleurs qu'interviennent du tout les mécanismes évoqués ci-dessus. Cette situation nous a conduits à utiliser, pour tenter d'identifier des molécules impliquées dans la cytotoxicité, des approches ouvertes, aussi indépendantes que possible d'idées préconçues quant au mécanisme en jeu. Une première approche fait appel à une méthodologie de biologie moléculaire. Elle repose sur le postulat 


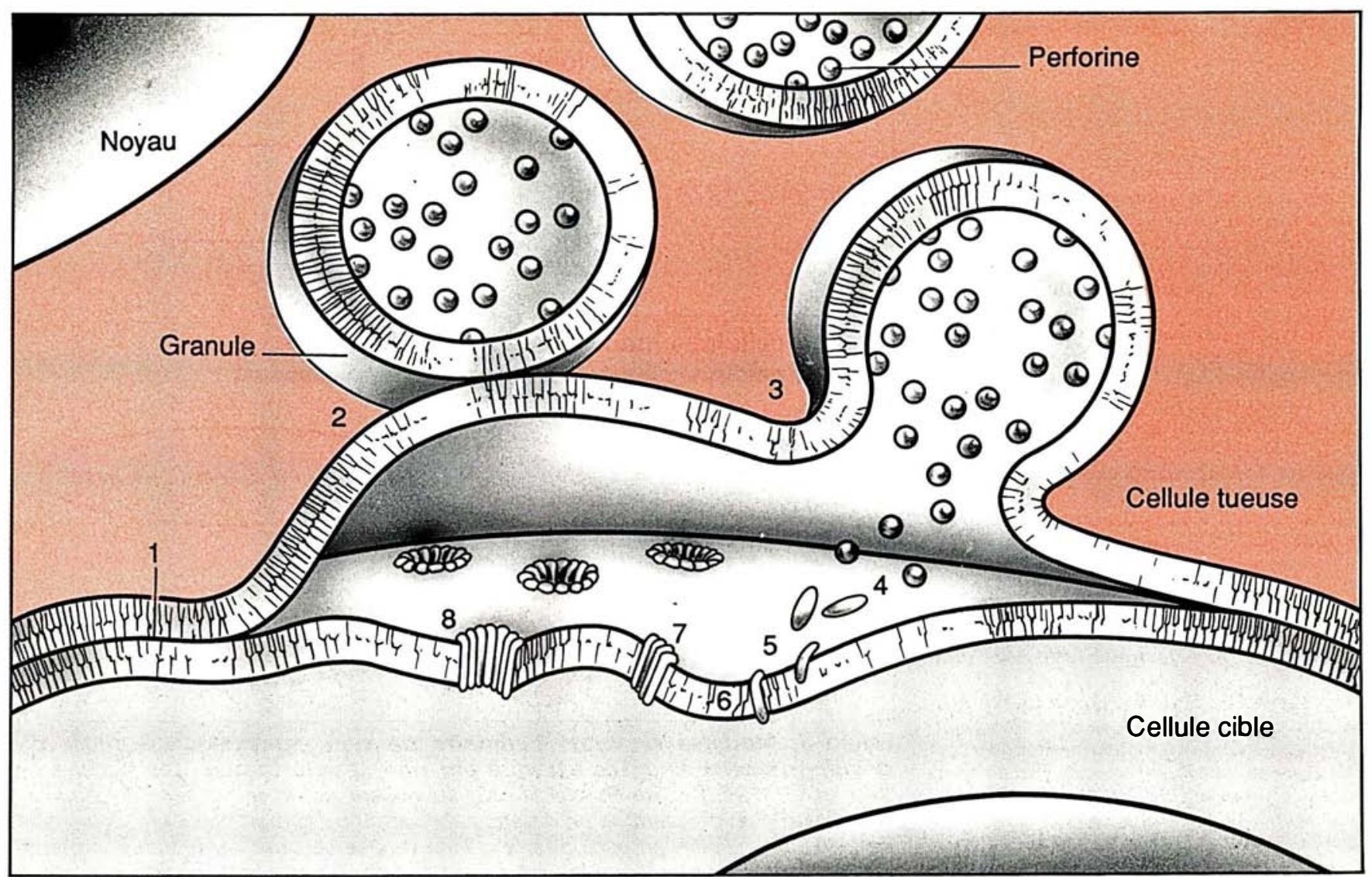

Figure 2. Modèle de type "exocytose granulaire/perforine" pour la lyse d'une cellule cible par une cellule tueuse. La cellule tueuse interagit avec sa cible (1), ce qui provoque (par l'intermédiaire, le cas échéant, du récepteur pour l'antigène et du complexe CD3) une « activation „ marquée par une augmentation du Ca++ cytosolique, une exocytose (2, 3) et la libération de la perforine contenue dans les granules de sécrétion (4). En présence de Ca+t, la perforine crée des pores dans la membrane plasmique de la cible $(5,6,7,8)$ qui, probablement à la suite de mouvements d'eau et de sels, meurt. (D'après Young JD et Cohn ZA. Scientific American 1988; 258: 28).

que des molécules impliquées dans une fonction peuvent être exprimées préférentiellement dans des cellules exerçant cette fonction. Nous avons ainsi essayé d'identifier des molécules exprimées seulement dans des cellules $\mathrm{T}$ cytotoxiques et non dans d'autres cellules (glissant ainsi d'un problème fonctionnel à un problème de différenciation ; et nous imposant, si des molécules exprimées préférentiellement dans des cellules $\mathrm{T}$ cytotoxiques sont trouvées, le trajet de retour vers la recherche de leur implication fonctionnelle). En pratique, cette approche implique la préparation par hybridation soustractive d'ADNc représentant les ARN messagers présents dans des cellules $\mathrm{T}$ cytotoxiques, diminués de ceux qui sont également présents dans des lymphocytes $\mathrm{B}$, puis le clonage de cet ADNc soustrait. La banque soustraite «TC-B» ainsi obtenue a $\mathrm{m} / \mathrm{s} n^{\circ} 8$ vol. 5 , octobre 89 ensuite été soumise à un criblage différentiel avec de l'ADNc radiomarqué provenant de cellules $T$ cytotoxiques ou avec des ADNc radiomarqués provenant d'autres cellules, à la recherche de clones positifs avec le premier et négatifs avec les seconds [45].

Nous avons ainsi isolé quatre molécules correspondant à de tels clones d'ADNc, que nous avons appelées CTLA-1, CTLA-2, CTLA-3 et CTLA-4. Des clones d'ADNc pleine taille ont été obtenus pour chacune de ces quatre molécules. Leur séquence a été établie, les gènes correspondants ont été obtenus, et leur localisation chromosomique a été déterminée chez la souris et aussi dans certains cas chez l'homme.

CTLA-1 et CTLA-3 sont des sérineestérases particulières exprimées essentiellement dans des cellules $\mathrm{T}$ activées [46], mises en évidence égale- ment dans d'autres laboratoires à la même période et en utilisant la même approche [47, 48]; (voir aussi [49]), ce qui reflète l'abondance des transcrits correspondants dans ces cellules. Le rôle de ces sérineestérases est actuellement discuté, et l'hypothèse populaire de leur intervention dans une cascade homologue à la cascade du complément reste à démontrer.

CTLA-4 se trouve être un nouveau membre de la superfamille des immunoglobulines, plus précisément un membre de la sous-famille de domaines $\mathrm{V}$ isolés (comme par exemple Thy 1, CD8, CD28). A l'intérieur de cette sous-famille, CTLA4 est particulier par son expression inductible lors de l'activation lymphocytaire [50]. Par analogie avec d'autres membres de cette superfamille, CTLA-4 est peut-être impliqué dans un rôle d'adhésion et/ou 
40. Thorn RM, Henney CS. Studies on the mechanism of lymphocyte-mediated cytolysis. VI. A reappraisal of the requirement for protein synthesis during $T$ cell-mediated lysis. J Immunol 1976; 116 : 146-9.

41. Kerr JFR, Searle J, Harmon BV, Bishop CJ. Apoptosis. In : Potten CS, ed. Perspectives on Mammalian Cell Death. OxfordNew York-Tokyo: Oxford University Press, 1987 : 93-128.

42. Lancki DW, Kaper BP, Fitch FW. The requirements for triggering of lysis by cytolytic T lymphocyte clones. II. Cyclosporin A inhibits TCR-mediated exocytosis but only selectively inhibits TCR-mediated lytic activity by cloned CTL. J Immunol 1989; 142 : 416-24.

43. Mercep M, Noguchi PD, Ashwell JD. The cell cycle block and lysis of an activated $\mathrm{T}$ cell hybridoma are distinct processes with different $\mathrm{Ca}^{2+}$ requirements and sensitivity to cyclosporine A. J Immunol 1989; 142 : 408592.

44. Trenn G, Taffs R, Hohman R, Kincaid R, Shevach EM, Sitkovsky M. Biochemical characterization of the inhibitory effect of CsA on cytolytic T lymphocyte effector functions. I Immunol 1989; 142 : 3796-802.

45. Brunet JF, Denizot F, Golstein P. A differential molecular biology search for genes preferentially expressed in functional $\mathrm{T}$ lymphocytes: the CTLA genes. Immunol Rev $1988 ; 103: 21-36$

46. Brunet JF, Dossei, M, Denizot F, et al. The inducible cytotoxic-T-lymphocyte-associated gene transcript CTLA-l sequence and gene localization to mouse chromosome 14 . Nature 1986 ; 322 : 268-71.

47. Lobe CG, Finlay BB, Paranchych W, Paetkau VH, Bleackley RC. Novel serine proteases encoded by two cytotoxic $\mathrm{T}$ lymphocyte-specific genes. Science 1986 ; 232 : 858-61. 48. Gershenfeld HK, Weissman IL. Cloning of a cDNA for a $T$ cell-specific serine protease from a cytotoxic T lymphocyte. Science 1986 ; $232: 854-8$.

49. Jenne DE, Tschopp J. Granzymes, a family of serine proteases released from granules of cytolytic $T$ lymphocytes upon $T$ cell receptor stimulation. Immunological Rev $1988 ; 103: 53-71$.

50. Brunet JF, Denizot F, Luciani MF, et al. A new member of the immunoglobulin superfamily-CTLA-4. Nature $1987 ; 328: 267-70$. 51. Dariavach $P$, Mattéi MG, Golstein $P$, Lefranc MP. Human Ig superfamily CTLA4 gene : Chromosomal localization and identity of protein sequence between murine and human CTLA-4 cytoplasmic domains. Eur J Immunol 1988; 18 : 1901-5.

52. Denizot F, Brunet JF, Roustan $P$, et al. Novel structures CTLA-2 alpha and CTLA2 beta expressed in mouse activated $T$ cells and mast cells and homologous to cysteine proteinase proregions. Eur J Immunol 1989; $19: 631-5$

53. Conzelmann A, Corthézy P, Cianfriglia M, Silva A, Nabholz M. Hybrids between rat lymphoma and mouse $T$ cells with inducible cytolytic activity. Nature $1982 ; 298: 170$.

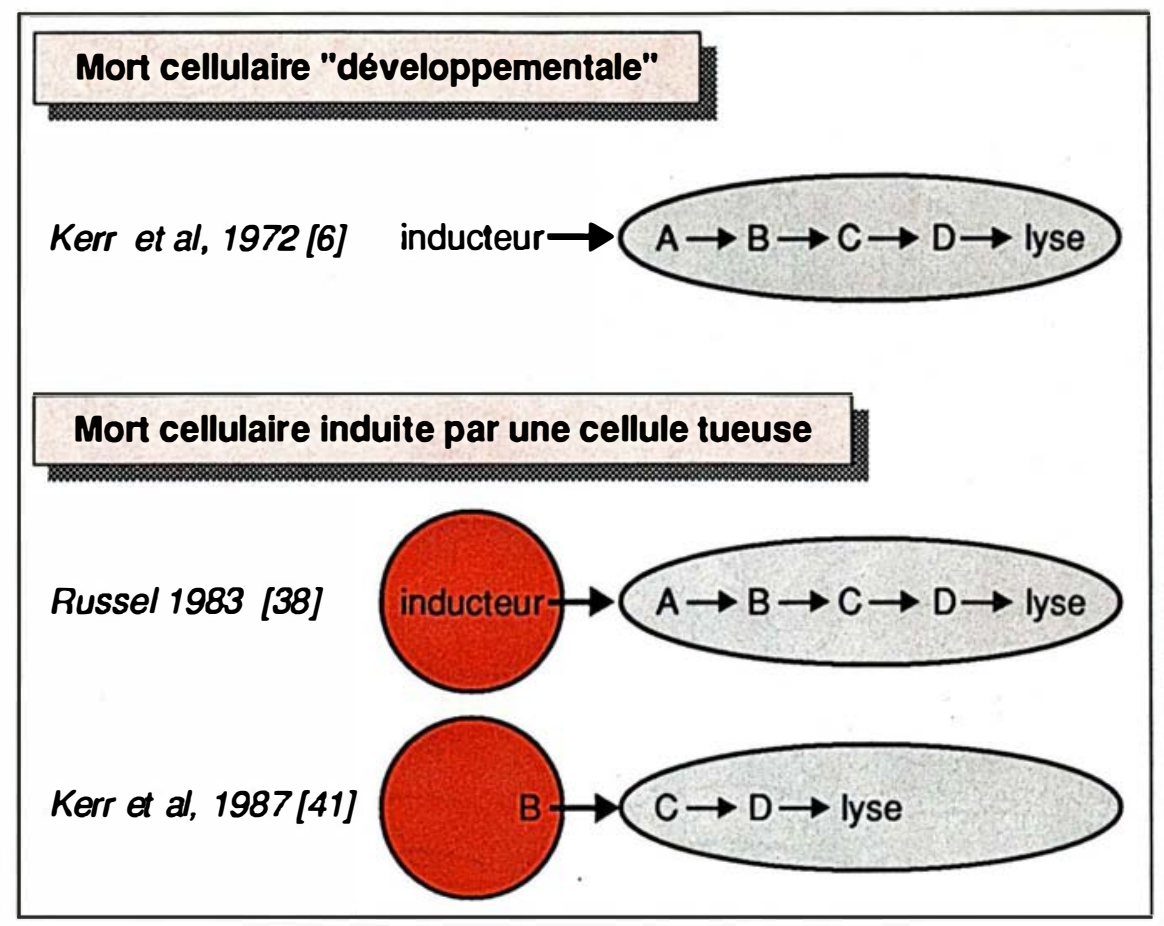

Figure 3. Modèles de mort cellulaire de type apoptose, au cours du développement et après attaque par une cellule tueuse. Les modèles de Kerr et al. (1972) et de Russel (1983) supposent qu'un inducteur déclenche la réalisation d'un programme en cascade aboutissant à la mort cellulaire. Le modèle de Kerr et al. (1987) propose que la cellule tueuse pourrait contribuer elle-même à la cascade en en réalisant les premières étapes ; les étapes suivantes, qui, après transfert de produits intermédiaires, seront poursuivies dans la cellule cible.

d'activation lymphocytaire. La probabilité de son importance fonctionnelle est renforcée par les données de séquence concernant non seulement CTLA-4 de souris mais également CTLA-4 humain [51]. D'une part, CTLA-4 présente une forte homologie avec la molécule d'activation CD28. D'autre part, les séquences protéiques des segments cytoplasmiques de CTLA-4 humain et murin sont identiques, alors qu'il existe de petites différences entre les séquences nucléiques correspondantes. Cette dernière observation montre que la pression en faveur de la conservation interspécifique de ce segment cytoplasmique s'exerce au niveau protéique, bon argument pour son importance fonctionnelle.

CTLA-2 est un homologue de prorégion de cystéine-protéase, exprimé lors de l'activation lymphocytaire [52]. CTLA-2 semble correspondre en fait à un module génétiquement autonome de pro-région enzymatique. Cette observation peut avoir un intérêt marqué en particulier en phylogenèse moléculaire. Il est tentant, une fois ces molécules isolées, de les étudier pour ellesmêmes indépendamment de la question initialement posée. Revenons-y néanmoins : ces molécules ont-elles un rôle, et lequel, dans des fonctions cellulaires $T$ ? Pour répondre à cette question, il nous faut disposer d'une prise sur les protéines correspondantes (étendant ainsi une approche expérimentale jusqu'alors limitée aux ARN et aux ADNc correspondants). Ceci implique en pratique l'obtention de peptides synthétiques ou de protéines de fusion à partir des ADNc, et l'utilisation de ces peptides ou de ces constructions pour l'immunisation d'animaux. Les anticorps anti-CTLA obtenus seront utilisés pour tenter d'activer ou d'inhiber des fonctions lymphocytaires T. Ce travail est actuellement en cours.

Une autre approche dans le laboratoire fait appel à des techniques de 
génétique somatique cellulaire. Nous sommes partis d'un hybridome cytotoxique, appelé PC60, développé par Markus Nabholz et al. à Lausanne [53]. Cet hybridome est interspécifique, résultant de la fusion d'une cellule tumorale de rat et d'une cellule lymphoïde cytotoxique de souris. Probablement du fait de cette interspécificité, cet hybridome est instable en culture, tendant à perdre sa cytotoxicité au bout de quelques semaines de culture in vitro. Nous avons tenté de prendre avantage de cette instabilité, de la façon suivante. Des cellules PC60 ont été mises en culture, et suivies quant à l'évolution de leur cytotoxicité. Au bout de quelques semaines, quand cette cytotoxicité diminue, les cellules sont clonées; parmi les différents clones obtenus, un clone cytotoxique est sélectionné et remis en culture pour un nouveau cycle. Quand la cytotoxicité de ce clone chute, il est luimême cloné, et de nouveau parmi les différents clones obtenus un clone cytotoxique est sélectionné. Plus d'une douzaine de tels sous-clonages en série ont été pratiqués sur une période totale de culture qui dépasse maintenant trois ans. Le but en est d'essayer d'émonder la cellule cytotoxique PC60 de structures non pertinentes à la lyse ou à la pousse. $\mathrm{La}$ réalité de la survenue d'un émondage a pu être démontrée, en collaboration avec Marie-Geneviève Mattéi à Marseille, sur la perte d'au moins une vingtaine de chromosomes depuis la mise en culture de cet hybridome, avec conservation de la fonction cytotoxique. Un émondage a pu être démontré également au niveau des transcrits, avec la perte en particulier des transcrits correspondant à la sérine-estérase CTLA-1. Cette perte dans une cellule qui reste cytotoxique permet d'exclure un rôle pour cette sérine-estérase dans le type de cytotoxicité exercé par cette cellule (Luciani et al., en préparation).

Ce système fonctionne donc en exclusion, permettant d'affirmer pour une molécule donnée sa nonimplication fonctionnelle, dans le cadre expérimental indiqué. Nous essayons actuellement d'obtenir des variants cytotoxiques négatifs de ces cellules, complémentables, ce qui nous permettrait de transformer cette approche d'exclusion en approche d'affirmation d'implication fonctionnelle de certaines molécules.

\section{Conclusion}

Nous avons évoqué ici, d'une part, les morts cellulaires développementales dans le système immunitaire et, d'autre part, les morts cellulaires induites par le système immunitaire. Ces deux catégories de mort cellulaire présentent au moins sur le plan morphologique un certain nombre de points communs, donnant ainsi lieu à une hypothèse unificatrice, quoiqu'il soit très possible que des ressemblances morphologiques soient sous-tendues par des différences mécanistiques. Ces dernières pourraient être révélées par une étude simultanée, comparative, de ces deux catégories de mort cellulaire.

Ces deux types de mort cellulaire ont par ailleurs des rapports de finalité complexes, organisés en succession ou en parallèle. En succession, si on met l'accent sur les finalités assez voisines de ces deux catégories de mort cellulaire dans le cadre du système immunitaire : la mort cellulaire développementale vise très schématiquement à sélectionner des lymphocytes capables de reconnaître le nonsoi ; ces lymphocytes sont dotés d'un mécanisme cytotoxique leur permettant de détruire les cellules présentant le non-soi, qu'ils ont été sélectionnés à reconnaître. En parallèle, si on veut bien prendre en considération le fait que, à la fois, cellules tueuses et mort développementale sont des instruments d'une lyse cellulaire finalisée à l'échelle de l'individu, pour le bien de cet individu

\section{Remerciements}

Les travaux du laboratoire ont bénéficié de la participation, en particulier, de J.F. Brunet, F. Denizot et M.F. Luciani et, plus récemment, de K. Harper, C. Balzano et E. Rouvier; de la collaboration, en particulier, de M.G. Mattéi (Inserm U. 242, Marseille) et de P. Dariavach et M.P. Lefranc (UA Cnrs 1191, Montpellier); du soutien du Cnrs et de l'Inserm, et également de l'ARC, de la FRM et du FEGEFLUC. Je remercie aussi mes collègues J. Davoust, C. Goridis et A.M. Schmitt-Verhulst pour leur relecture de ce manuscrit.

\section{Summary}

Cell death and the immune system

Cell death occurs in the immune system, as part of its normal development, through a process of elimination of lymphocytes bearing inappropriate receptors. This cell death is programmed, both as to its occurrence in space and time within the immune system, and as to its course within each cell, leading to the notions of apoptosis and of induced suicide, respectively. Cell death also occurs via the immune system, especially through the action of cytotoxic lymphocytes. The mechanism of cytotoxicity by the latter is still not known; several hypotheses have been raised, some of which take into account a degree of morphological similarity with the developmental cell death alluded to above. To identify molecules involved in cell-mediated cytotoxicity, in particular a molecular biology approach involving the differential screening of a subtracted cDNA library and a somatic cell genetic approach are being used.

\begin{tabular}{|c|}
\hline 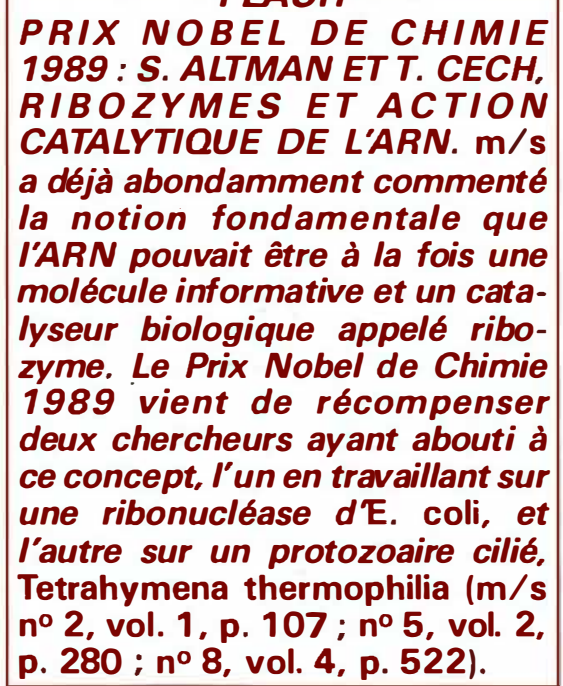 \\
\hline
\end{tabular}

TIRÉS A PART

P. Golstein 